\title{
Prognostic implications of resting distal coronary-to-aortic pressure ratio compared with fractional flow reserve: a 10- year follow-up study after deferral of revascularisation
}

\author{
G. W. M. Wijntjens · T. P. van de Hoef · M. Meuwissen · M. Echavarría-Pinto · T. Murai · V. E. Stegehuis · K. T. Koch • \\ S. A. Chamuleau · M. Voskuil · R. J. de Winter · J. G. P. Tijssen · J. J. Piek
}

Published online: 21 January 2020

(c) The Author(s) 2020

\begin{abstract}
Introduction The distal coronary-to-aortic pressure ratio $\left(P_{\mathrm{d}} / P_{\mathrm{a}}\right)$ is a non-hyperaemic physiological index to assess the functional severity of coronary stenoses. Studies comparing $P_{\mathrm{d}} / P_{\mathrm{a}}$ with fractional flow reserve (FFR) show superior diagnostic efficiency for myocardial ischaemia. Nevertheless, a direct comparison regarding long-term clinical outcomes is still not available. The present observational study compared the prognostic value of $P_{\mathrm{d}} / P_{\mathrm{a}}$ and FFR for major adverse cardiac events (MACE) during a 10-year follow-up period after deferral of revascularisation.

Methods Between April 1997 and September 2006, we evaluated 154 coronary stenoses (154 patients) in which revascularisation was deferred with intracoronary pressure and flow measurements during the rest-
\end{abstract}

G.W.M. Wijntjens and T.P. van de Hoef contributed equally

Electronic supplementary material The online version of this article (https://doi.org/10.1007/s12471-020-01365-6) contains supplementary material, which is available to authorized users.

G. W. M. Wijntjens · T. P. van de Hoef $(\varangle) \cdot$ T. Murai •

V. E. Stegehuis · K. T. Koch · S. A. Chamuleau · R. J. de Winter ·

J. G. P. Tijssen · J. J. Piek

Heart Centre, Amsterdam Universitair Medische Centra,

locatie Academic Medical Centre, Amsterdam, The

Netherlands

t.p.vandehoef@amsterdamumc.nl

M. Meuwissen

Department of Cardiology, Amphia Hospital, Breda, The Netherlands

\section{Echavarría-Pinto}

Hospital General ISSSTE, Facultad de Medicina, Universidad Autónoma de Querétaro, Querétaro, Mexico

M. Voskuil

Department of Cardiology, University Medical Centre Utrecht, Utrecht, The Netherlands ing and hyperaemic state. Long-term follow-up (median: 11.8 years) was performed to document the occurrence of MACE, defined as a composite of cardiac death, myocardial infarction and target vessel revascularisation.

Results The study population comprised angiographically intermediate coronary stenoses, with a mean diameter stenosis of $53 \pm 8 \%$, and intermediate physiological severity with a median FFR of 0.82 (Q1, Q3: $0.76,0.88)$. The association of $P_{\mathrm{d}} / P_{\mathrm{a}}$ with long-term MACE was similar to that of FFR [FFR-standardised hazard ratio (sHR): 0.77, 95\% confidence interval (CI): 0.61-0.98; $P_{\mathrm{d}} / P_{\mathrm{a}}$-sHR: 0.80, 95\% CI: 0.67-0.96]. In the presence of disagreement between $P_{\mathrm{d}} / P_{\mathrm{a}}$ and FFR, normal $P_{\mathrm{d}} / P_{\mathrm{a}}$ was generally associated with high coronary flow reserve (CFR) and a favourable clinical outcome, whereas abnormal $P_{\mathrm{d}} / P_{\mathrm{a}}$ was generally associated with CFR around the ischaemic cut-point and an impaired clinical outcome, regardless of the accompanying FFR value.

Conclusion The present study suggests that $P_{\mathrm{d}} / P_{\mathrm{a}}$ provides at least equivalent prognostic value compared with FFR. When $P_{\mathrm{d}} / P_{\mathrm{a}}$ disagreed with FFR, the base-

\section{What's new?}

- The distal coronary-to-aortic pressure ratio $\left(P_{\mathrm{d}} / P_{\mathrm{a}}\right)$ demonstrates a continuous and independent relationship with subsequent long-term clinical outcomes.

- The prognostic value of $P_{\mathrm{d}} / P_{\mathrm{a}}$ is at least equivalent to that of fractional flow reserve (FFR), but exceeds FFR as a risk stratification tool at their contemporary clinical cut-off values.

- When $P_{\mathrm{d}} / P_{\mathrm{a}}$ disagreed with FFR, $P_{\mathrm{d}} / P_{\mathrm{a}}$ conferred superior prognostic value in this study population. 
line index conferred superior prognostic value in this study population.

Keywords Coronary artery disease $\cdot$ Fractional flow reserve - Resting distal-to-aortic pressure ratio Major adverse cardiac events

\section{Introduction}

Guidelines on coronary revascularisation recommend the hyperaemia-dependent fractional flow reserve (FFR) for clinical decision-making, yet its clinical adoption has remained limited. As a corollary, the resting distal coronary-to-aortic pressure ratio $\left(P_{\mathrm{d}} / P_{\mathrm{a}}\right)$ and the instantaneous wave-free ratio (iFR) were (re)introduced with the aim of simplifying the use of coronary physiology in daily clinical practice by enabling its assessment during resting conditions [1-3]. Although baseline indices and FFR show equal diagnostic accuracy for positron emission tomographyderived flow abnormalities [4, 5], studies using invasive Doppler-derived coronary flow reserve (CFR) as the reference standard documented superior identification of flow abnormalities by $P_{\mathrm{d}} / P_{\mathrm{a}}$ and iFR over FFR [3, 6, 7]. Doppler-derived CFR is considered a critical determinant of myocardial ischaemia and has relevant prognostic value in the setting of stable ischaemic heart disease [8, 9]. Accordingly, two landmark randomised clinical trials demonstrated that an iFR-guided revascularisation strategy is non-inferior to a FFR-guided revascularisation strategy in terms of clinical outcome at 1-year follow-up [10, 11]. However, the long-term prognostic value of $P_{\mathrm{d}} / P_{\mathrm{a}}$ has yet not been compared to that of FFR. Therefore, the aim of the present study was to compare the prognostic value of $P_{\mathrm{d}} / P_{\mathrm{a}}$, with that of FFR for major adverse cardiac events (MACE) during a 10-year follow-up period after deferral of revascularisation.

\section{Methods}

\section{Data source}

Between April 1997 and September 2006, we prospectively enrolled patients with angina referred for evaluation of $\geq 1$ intermediate coronary stenosis $(40 \%-70 \%$ diameter stenosis at visual assessment) in a series of study protocols [12-14] that included intracoronary pressure and flow measurements during resting and hyperaemic conditions. We excluded patients with ostial stenoses, serial stenoses, severe renal function impairment, significant left main stenosis, atrial fibrillation, myocardial infarction $<6$ weeks before screening, prior coronary artery bypass surgery (CABG), or visible collateral development to the perfusion territory of interest. The study protocols complied with the Declaration of Helsinki and were approved by the institutional ethics committee. All patients gave written informed consent.

\section{Study procedures and subsequent treatment}

Coronary angiography was performed according to standard practice. Quantitative coronary angiography analyses were performed offline using validated software (QCA-CMS version 3.32, MEDIS, Leiden, The Netherlands). Intracoronary nitroglycerin $(0.1 \mu \mathrm{g})$ was given prior to invasive measurements. Intracoronary pressure and flow was measured with $0.014^{\prime \prime}$ pressure and Doppler sensor-equipped guide wires (PhilipsVolcano, San Diego, CA, USA) during resting and hyperaemic conditions. Hyperaemia was induced by an intracoronary bolus of adenosine (20-40 $\mu \mathrm{g})$. Revascularisation was performed at the operator's discretion, and decisions on further treatment and medication during follow-up were entirely left to the discretion of the treating cardiologist $[8,15]$.

\section{Long-term follow-up}

Three-, 6-, 12-month and long-term follow-up was performed by clinical visit or telephone contact to document the occurrence of MACE, defined as the composite of cardiac death, acute myocardial infarction attributable to the target vessel, and clinically driven revascularisation of the target-vessel by CABG or percutaneous coronary intervention (PCI). All patient-reported events were verified and adjudicated after evaluating hospital records or contacting the treating physicians.

\section{Haemodynamic data analysis}

The physiological indices were defined as:

- $\mathrm{FFR}=$ mean $P_{\mathrm{d}} /$ mean $P_{\mathrm{a}}$ (hyperaemia)

- $P_{\mathrm{d}} / P_{\mathrm{a}}=$ mean $P_{\mathrm{d}} /$ mean $P_{\mathrm{a}}$ (resting conditions)

- $\mathrm{CFR}=$ hyperaemic average peak flow velocity (APV) / baseline APV

For dichotomous evaluations, FFR $\leq 0.80$ [16], and $P_{\mathrm{d}} / P_{\mathrm{a}} \leq 0.92$ [2] were considered abnormal (Fig. 1).

\section{Statistical analysis}

In the presence of multiple stenoses of intermediate severity, one was randomly marked the index stenosis and used for subsequent analyses. Normality of the distribution and homogeneity of variances were tested with the Shapiro-Wilk statistic and Levene's test, respectively. Continuous variables are presented as mean \pm standard deviation or median [1st, 3rd quartile (Q1, Q3)], and compared with Student $t$-test or Mann-Whitney U-test. Categorical variables are presented as frequency (percentage) and compared with Fisher exact test. The prognostic value of FFR and $P_{\mathrm{d}} / P_{\mathrm{a}}$ for 10-year MACE was assessed using Cox regression analyses, adjusted for the effect of relevant clinical characteristics. The best-fit model for adjustment was identified using Akaike's information 


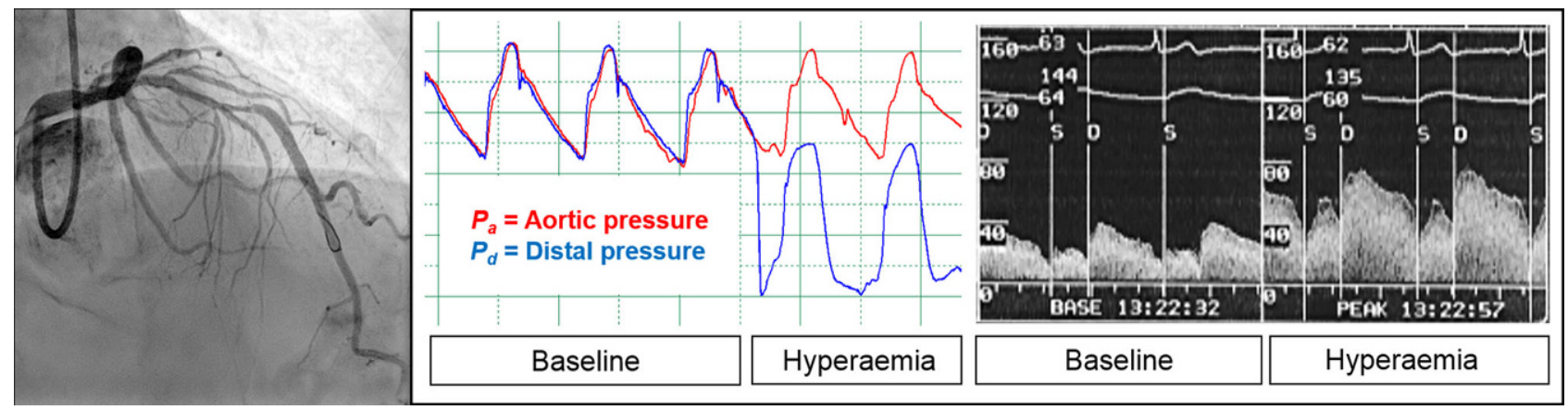

Fig. 1 Example of intracoronary pressure and flow measurements. Resting distal-to-aortic pressure ratio $\left(P_{\mathrm{d}} / P_{\mathrm{a}}\right)$ is defined as the distal coronary pressure to aortic pressure ratio during baseline conditions. Fractional flow reserve is defined as the distal coronary pressure to aortic pressure ratio during hyper- aemic conditions induced by hyperaemic agents. Coronary flow reserve is defined as the coronary flow velocity during hyperaemia to coronary flow velocity during baseline ratio measured distal from the stenosis of interest
Table 1 Demographic, angiographic and physiological characteristics

\begin{tabular}{|c|c|c|}
\hline Number & & $n=154$ \\
\hline \multicolumn{3}{|c|}{ Demographics } \\
\hline & Age, years & $61 \pm 11$ \\
\hline & Male & $110(71)$ \\
\hline \multicolumn{3}{|c|}{ Risk factors for coronary artery disease } \\
\hline & Hypertension & $60(39)$ \\
\hline & Hyperlipidaemia & $89(58)$ \\
\hline & Positive family history & $76(49)$ \\
\hline & Cigarette smoking & $48(31)$ \\
\hline & Diabetes mellitus & $24(16)$ \\
\hline & Prior myocardial infarction & $57(37)$ \\
\hline & Prior $\mathrm{PCl}$ & $34(22)$ \\
\hline \multicolumn{3}{|c|}{ Medication at hospital admission } \\
\hline & Beta-blocker & $120(78)$ \\
\hline & Nitrates & $110(71)$ \\
\hline & Calcium antagonists & $101(66)$ \\
\hline & ACE inhibitors & $28(18)$ \\
\hline & Statins & $87(56)$ \\
\hline & Acetylsalicylic acid & $149(97)$ \\
\hline \multicolumn{3}{|c|}{ Angiographic characteristics } \\
\hline & Reference vessel diameter, $\mathrm{mm}$ & $2.9 \pm 0.6$ \\
\hline & Diameter stenosis, $\%$ & $53 \pm 8$ \\
\hline & Minimal lumen diameter & $1.4 \pm 0.4$ \\
\hline \multicolumn{3}{|c|}{ Physiological characteristics } \\
\hline & FFR & $0.82(0.76,0.88)$ \\
\hline & FFR when FFR $\leq 0.80(n=67)$ & $0.74(0.70,0.78)$ \\
\hline & FFR when FFR > $0.80(n=87)$ & $0.88(0.84,0.92)$ \\
\hline & $P_{\mathrm{d}} / P_{\mathrm{a}}$ & $0.94(0.91,0.97)$ \\
\hline & $P_{\mathrm{d}} / P_{\mathrm{a}}$ when $P_{\mathrm{d}} / P_{\mathrm{a}} \leq 0.92(n=53)$ & $0.90(0.86,0.91)$ \\
\hline & $P_{\mathrm{d}} / P a$ when $P_{\mathrm{d}} / P_{\mathrm{a}}>0.92(n=101)$ & $0.97(0.94,0.98)$ \\
\hline & \begin{tabular}{l|l} 
CFR \\
\end{tabular} & $2.5(2.1,2.9)$ \\
\hline & CFR when CFR < $2.0(n=31)$ & $1.8(1.5,1.9)$ \\
\hline & CFR when CFR $\geq 2.0(n=123)$ & $2.6(2.3,3.1)$ \\
\hline
\end{tabular}




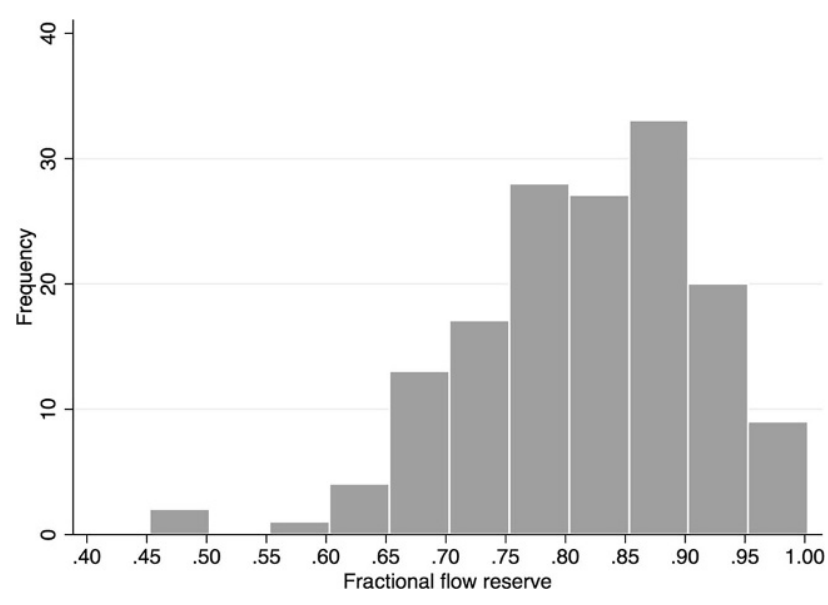

Fig. 2 Distribution of fractional flow reserve values across the study population

criterion, where candidate covariates were: clinical characteristics (Tab. 1) and the interrogated vessel. All Cox proportional-hazards models were preceded by verification of the proportional hazard assumption using Schoenfeld's residuals. Results are presented as standardised hazard ratios (sHRs) and their 95\% confidence intervals (CIs), estimated from the Cox proportional-hazard models by exponentiating the $\beta$-coefficient multiplied by the $\operatorname{SD}[\exp (\beta \times \mathrm{SD})]$. Next, according to their clinical cut-off values, 10-year MACE rates for normal and abnormal FFR and $P_{\mathrm{d}} / P_{\mathrm{a}}$ were estimated using the Kaplan-Meier (KM) method, and differences were tested with the Wilcoxon-Breslow-Gehan test of equality (Breslow $p$ ). Finally, KM estimates of 10-year MACE were evaluated across the four quadrants of $P_{\mathrm{d}} / P_{\mathrm{a}}$ and FFR (dis)agreement, where differences were evaluated with Breslow $p$. A $p$ value below the 2 -sided $\alpha$-level of 0.05 was considered statistically significant. The STATA 13.1 statistical software package (StataCorp, College Station, TX, USA) was used for all calculations.

\section{Results}

\section{Patient population}

A total of 228 patients (299 stenoses) were included. Revascularisation was deferred in 159 patients, in 154 of whom (183 stenoses) resting and hyperaemic coronary pressure and flow velocity data and long-term follow-up were available. In patients with multiple stenoses, one was chosen at random for MACE analyses, which consequently included 154-patients with 154 stenoses. Median follow-up in these patients was 11.8 years (Q1, Q3: 10.0, 13.3 years). Mean age was $60 \pm 11$ years and $71 \%$ of patients were male. Additional baseline characteristics are depicted in Tab. 1.

\section{Angiographic and physiological measurements}

The study population comprised angiographically intermediate stenoses, with a mean diameter stenosis of $53 \pm 8 \%$, and intermediate physiological severity with a median FFR of 0.82 (Q1, Q3: 0.76, 0.88) (Fig. 2), median $P_{\mathrm{d}} / P_{\mathrm{a}}$ of 0.94 (Q1, Q3: 0.91, 0.97) and median CFR of 2.5 (Q1, Q3: 2.1, 2.9). Stenoses were haemodynamically significant in $43.5 \%$ and $34.4 \%$ of cases according to FFR and $P_{\mathrm{d}} / P_{\mathrm{a}}$, respectively. Angiographic and physiological characteristics are depicted in Tab. 1.

\section{Clinical outcome after deferral of revascularisation stratified by FFR and $P_{d} / P_{a}$}

The best-fit model for adjustment included angiotensin-converting-enzyme inhibitor use, the presence of diabetes mellitus and age. Cox regression analysis adjusted for these variables demonstrated that FFR and $P_{\mathrm{d}} / P_{\mathrm{a}}$ were both significantly and equivalently associated with long-term MACE [FFR-sHR: 0.77 (95\% CI: 0.61-0.98), $p=0.033 ; P_{\mathrm{d}} / P_{\mathrm{a}}$-sHR: 0.80 (95\% CI: 0.67-0.96), $p=0.014$ ] (Tab. 2). Fig. 3 shows the KM curves for cumulative MACE up to 10 years of follow-up according to normal or abnormal results for FFR and $P_{\mathrm{d}} / P_{\mathrm{a}}$. At 10-year follow-up, the KM estimate of MACE was not significantly different for stenoses with abnormal versus normal FFR (FFR $\leq 0.80$ : $45.8 \%$ vs FFR $>0.80$ : $34.4 \%$; Breslow $p=0.17$ : Fig. 3a). In contrast, the KM estimate of MACE at 10-year followup for stenoses with abnormal $P_{\mathrm{d}} / P_{\mathrm{a}}$ was significantly higher than for stenoses with normal $P_{\mathrm{d}} / P_{\mathrm{a}}$ $\left(P_{\mathrm{d}} / P_{\mathrm{a}} \leq 0.92: 52.0 \%\right.$ vs $P_{\mathrm{d}} / P_{\mathrm{a}}>0.9232 .7 \%$; Breslow $p=0.006$ : Fig. 3b).

\section{Clinical outcome according to discordance between} FFR and $P_{d} / P_{a}$

$P_{\mathrm{d}} / P_{\mathrm{a}}$ agreed with FFR in $71 \%$ of stenoses (110 out of 154 ), of which $25 \%$ of the stenoses (38 out of 154)

Table 2 Univariate and adjusted Cox regression analyses for long-term major adverse cardiac events

\begin{tabular}{|c|c|c|c|c|c|}
\hline \multicolumn{6}{|c|}{ Overall study population ( $n=154)$} \\
\hline \multicolumn{3}{|c|}{ Univariate analysis } & \multicolumn{3}{|c|}{ Adjusted analysis $^{\mathrm{a}}$} \\
\hline Variable & $\operatorname{sHR}(95 \% \mathrm{Cl})$ & $p$-value & Variable & $\operatorname{sHR}(95 \% \mathrm{Cl})$ & $p$-value \\
\hline$P_{\mathrm{d}} / P_{\mathrm{a}}$ & $0.76(0.63-0.91)$ & 0.003 & $P_{\mathrm{d}} / P_{\mathrm{a}}$ & $0.80(0.67-0.96)$ & 0.014 \\
\hline FFR & $0.76(0.59-0.98)$ & 0.034 & FFR & $0.77(0.61-0.98)$ & 0.033 \\
\hline
\end{tabular}




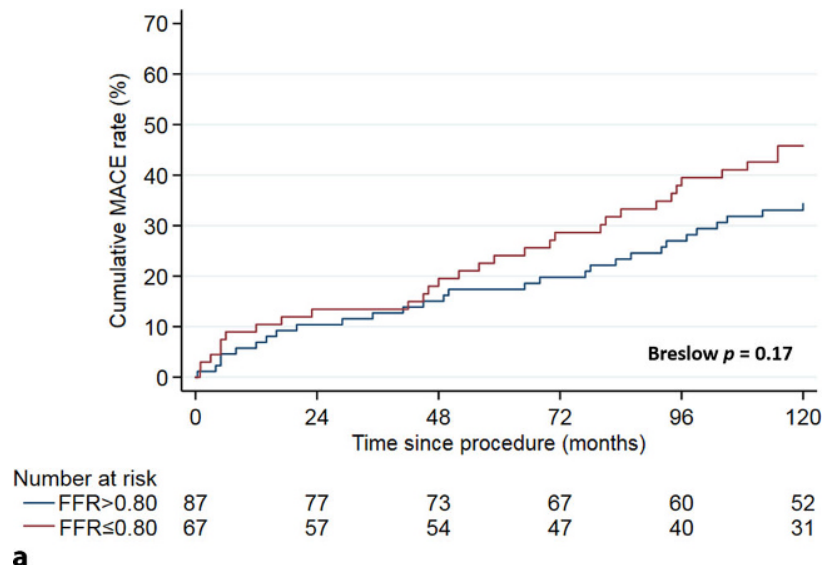

a

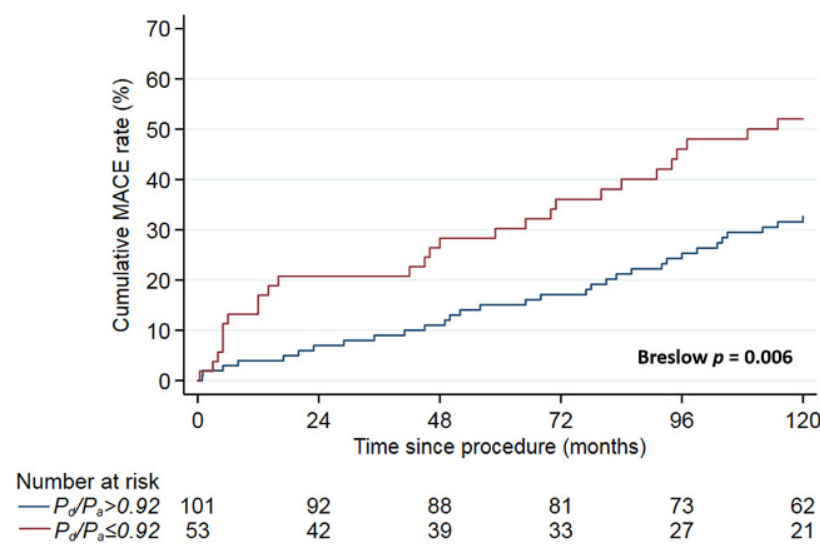

b

Fig. 3 Kaplan-Meier estimates of major adverse cardiac event (MACE) rate during 10 years of follow-up, stratified by: a fractional flow reserve $(F F R) ; \mathrm{FFR} \leq 0.80$ was considered abnormal. b Resting distal coronary-to-aortic pressure ratio $\left(P_{\mathrm{d}} / P_{\mathrm{a}}\right) . P_{\mathrm{d}} / P_{\mathrm{a}} \leq 0.92$ was considered abnormal

were concordant abnormal, and $47 \%$ of stenoses (72 out of 154) were concordant normal. $P_{\mathrm{d}} / P_{\mathrm{a}}$ disagreed with FFR in $29 \%$ of stenoses (44 out of 154), and was characterised by abnormal FFR and normal $P_{\mathrm{d}} / P_{\mathrm{a}}$ in $19 \%$ of stenoses (29 out of 154) and by normal FFR and abnormal $P_{\mathrm{d}} / P_{\mathrm{a}}$ in $10 \%$ of stenoses (15 out of 154 ) (Fig. 4a). Fig. 4b depicts the KM curves for cumulative MACE up to 10-year follow-up stratified by $P_{\mathrm{d}} / P_{\mathrm{a}}$ and FFR agreement. A normal $P_{\mathrm{d}} / P_{\mathrm{a}}$ was generally associated with a favourable clinical outcome, regardless of the accompanying FFR value, whereas abnormal $P_{\mathrm{d}} / P_{\mathrm{a}}$ was generally associated with an impaired clinical outcome, regardless of the accompanying FFR value (overall Breslow $p=0.04$ ).

\section{Agreement with CFR}

CFR was significantly different across the four quadrants of $P_{\mathrm{d}} / P_{\mathrm{a}}$ and FFR (dis)agreement (Tab. 3). A normal $P_{\mathrm{d}} / P_{\mathrm{a}}$ was generally associated with high CFR and an abnormal $P_{\mathrm{d}} / P_{\mathrm{a}}$ was generally associated with CFR values around the ischaemic cut-off, regardless of the accompanying FFR value (overall $p=0.006$ ).
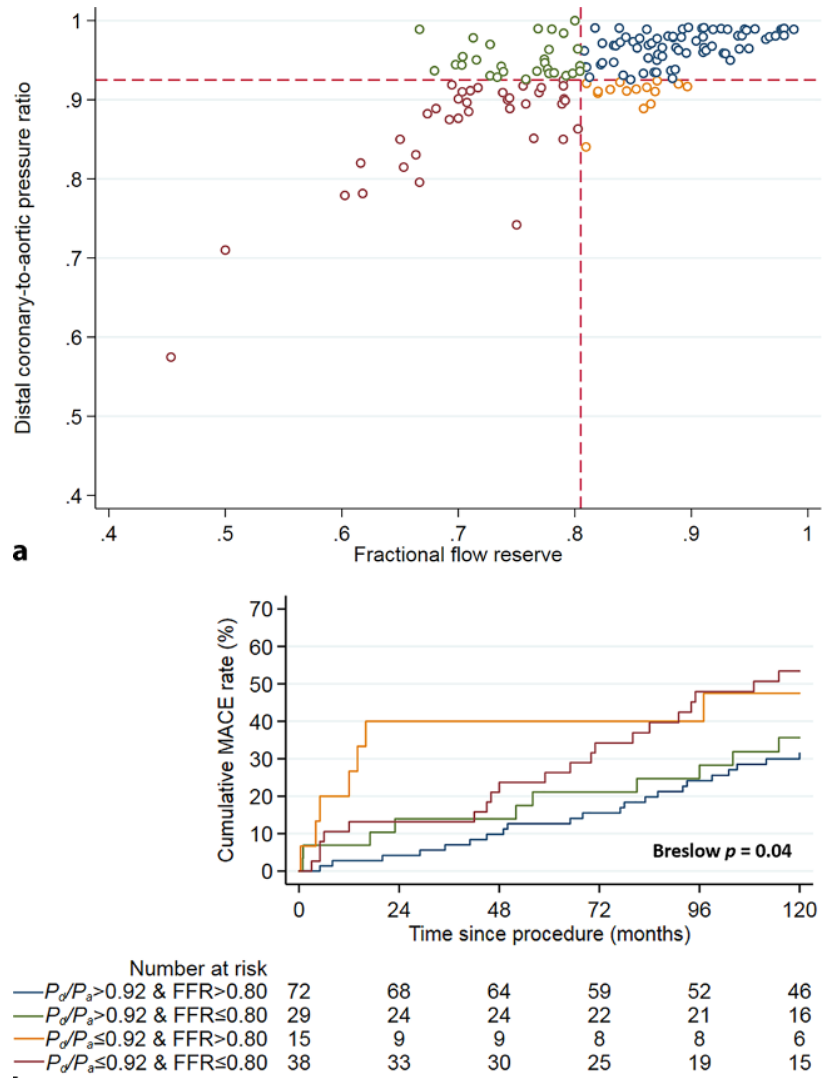
b

Fig. 4 a, b Discordance between resting distal coronary to aortic pressure ratio $\left(P_{\mathrm{d}} / P_{\mathrm{a}}\right)$ and fractional flow reserve (FFR). a Scatterplot of $P_{\mathrm{d}} / P_{\mathrm{a}}$ and FFR. The dotted lines represent the respective cut-off values for $P_{\mathrm{d}} / P_{\mathrm{a}}(0.92)$, and FFR (0.80). b Kaplan-Meier estimates of major adverse cardiac event (MACE) rate during 10 years of follow-up, stratified by $P_{\mathrm{d}} / P_{\mathrm{a}}$-FFR discordance

\section{Discussion}

The current article is the first to document that $P_{\mathrm{d}} / P_{\mathrm{a}}$ demonstrates a continuous and independent relationship with subsequent long-term clinical outcomes which is at least equivalent to that of FFR, and that $P_{\mathrm{d}} / P_{\mathrm{a}}$ exceeds FFR as a risk stratification tool at the contemporary clinical cut-off values. When discordance with FFR occurs, $P_{\mathrm{d}} / P_{\mathrm{a}}$ may therefore confer superior clinical value.

\section{Prognostic relevance of resting versus hyperaemic stenosis pressure drops}

The superior prognostic relevance of resting $P_{\mathrm{d}} / P_{\mathrm{a}}$ over FFR at contemporary cut-offs may be explained by a better agreement of $P_{\mathrm{d}} / P_{\mathrm{a}}$ with coronary flow. Our observations support those of previous studies that demonstrated a better relationship between resting indices and Doppler-derived CFR than for FFR and Doppler-derived CFR [3, 6, 7]. It has been documented that, when FFR disagrees with CFR, CFR has superior prognostic value over FFR for long-term 
Table 3 Study population characteristics according to FFR $-P_{\mathrm{d}} / P_{\mathrm{a}}$ disagreement

\begin{tabular}{|c|c|c|c|c|c|c|}
\hline & $\begin{array}{l}\text { FFR }>0.80 \text { and } \\
P_{\mathrm{d}} P_{\mathrm{d}} / P_{\mathrm{a}}>0.92\end{array}$ & \multicolumn{2}{|c|}{$\mathrm{FFR} \leq 0.80$ and $P_{\mathrm{d}} / P_{\mathrm{a}}>0.92$} & $\begin{array}{l}\text { FFR }>0.80 \text { and } \\
P_{\mathrm{d}} / P_{\mathrm{a}} \leq 0.92\end{array}$ & $\begin{array}{l}\mathrm{FFR} \leq 0.80 \text { and } \\
P_{\mathrm{d}} / P_{\mathrm{a}} \leq 0.92\end{array}$ & $\begin{array}{l}\text { Overall } \\
p \text {-value }\end{array}$ \\
\hline Number of patients & 72 & \multicolumn{2}{|l|}{29} & 15 & 38 & \\
\hline \multicolumn{7}{|l|}{ Demographics } \\
\hline Age, years & $61 \pm 11$ & \multicolumn{2}{|l|}{$57 \pm 11$} & $66 \pm 8$ & $60 \pm 11$ & 0.09 \\
\hline Male gender, $n(\%)$ & $51(71)$ & \multicolumn{2}{|l|}{$22(76)$} & $7(47)$ & $30(78)$ & 0.14 \\
\hline \multicolumn{7}{|l|}{ Coronary risk factors } \\
\hline Hypertension, $n(\%)$ & $43(60)$ & \multicolumn{2}{|l|}{$8(28)$} & $9(60)$ & $14(37)$ & 0.22 \\
\hline Hyperlipidaemia, $n(\%)$ & $41(57)$ & \multicolumn{2}{|l|}{$17(59)$} & $8(53)$ & $23(61)$ & 0.97 \\
\hline $\begin{array}{l}\text { Positive family history, } \\
n(\%)\end{array}$ & $33(46)$ & \multicolumn{2}{|l|}{$14(48)$} & $9(60)$ & $20(53)$ & 0.76 \\
\hline Cigarette smoking, $n(\%)$ & $20(28)$ & \multicolumn{2}{|l|}{$9(31)$} & $2(13)$ & $17(45)$ & 0.13 \\
\hline Diabetes mellitus, $n(\%)$ & $9(13)$ & \multicolumn{2}{|l|}{$8(28)$} & $3(20)$ & $4(11)$ & 0.2 \\
\hline $\begin{array}{l}\text { Prior myocardial infarc- } \\
\text { tion, } n(\%)\end{array}$ & $33(46)$ & \multicolumn{2}{|l|}{$8(28)$} & $3(20)$ & $13(34)$ & 0.15 \\
\hline Prior PCl, $n(\%)$ & $20(28)$ & \multicolumn{2}{|l|}{$8(28)$} & $2(13)$ & $4(11)$ & 0.14 \\
\hline \multicolumn{7}{|l|}{ Medication at hospital admission } \\
\hline Beta-blocker, $n(\%)$ & $57(79)$ & \multicolumn{2}{|l|}{$24(83)$} & $10(67)$ & $29(76)$ & 0.63 \\
\hline Nitrates, $n(\%)$ & $51(71)$ & \multicolumn{2}{|l|}{$19(66)$} & $12(80)$ & $28(74)$ & 0.8 \\
\hline $\begin{array}{l}\text { Calcium antagonists, } \\
n(\%)\end{array}$ & $44(61)$ & \multicolumn{2}{|l|}{$17(59)$} & $11(73)$ & $29(76)$ & 0.31 \\
\hline ACE inhibitors, $n(\%)$ & $15(21)$ & \multicolumn{2}{|l|}{$2(7)$} & $2(13)$ & $9(24)$ & 0.28 \\
\hline $\begin{array}{l}\text { Lipid-lowering drugs, } \\
n(\%)\end{array}$ & $39(54)$ & \multicolumn{2}{|l|}{$15(52)$} & $9(60)$ & $24(63)$ & 0.77 \\
\hline Acetylsalicylic acid, $n(\%)$ & $69(96)$ & \multicolumn{2}{|l|}{$29(100)$} & $15(100)$ & $36(95)$ & 0.76 \\
\hline \multicolumn{7}{|l|}{ Angiographic characteristics } \\
\hline Diameter stenosis, $\%$ & $53(44-57)$ & & $55(50-62)$ & $51(47-56)$ & $55(50-58)$ & 0.46 \\
\hline Reference diameter, $\mathrm{mm}$ & $3.0(2.6-3.5)$ & & $2.7(2.5-3.3)$ & $2.8(2.5-3.0)$ & $2.6(2.3-3.1)$ & 0.09 \\
\hline $\begin{array}{l}\text { Minimal lumen diameter, } \\
\mathrm{mm}\end{array}$ & $1.5(1.1-1.7)$ & & $1.2(1.1-1.6)$ & $1.3(1.1-1.5)$ & $1.2(1.1-1.3)$ & 0.03 \\
\hline \multicolumn{7}{|l|}{ Physiological characteristics } \\
\hline APV basal, $\mathrm{cm} / \mathrm{s}$ & $15(12-18)$ & & $18(13-24)$ & $16(13-24)$ & $17(11-21)$ & 0.19 \\
\hline APV hyperaemia, $\mathrm{cm} / \mathrm{s}$ & $38(31-48)$ & & $38(31-55)$ & $38(29-54)$ & $36(30-45)$ & 0.68 \\
\hline CFR & $2.6(2.2-3.0)$ & & $2.5(1.9-2.9)$ & $2.2(1.8-2.8)$ & $2.2(1.8-2.7)$ & 0.006 \\
\hline FFR & $0.89(0.85-0.93)$ & & $\begin{array}{l}0.78 \\
(0.73-0.78)\end{array}$ & $0.85(0.82-0.87)$ & $0.71(0.67-0.76)$ & 0.001 \\
\hline$P_{\mathrm{d}} / P_{\mathrm{a}}$ & $0.97(0.96-0.98)$ & & $\begin{array}{l}0.94 \\
(0.94-0.96)\end{array}$ & $0.91(0.91-0.92)$ & $0.89(0.85-0.91)$ & 0.001 \\
\hline
\end{tabular}

clinical outcomes $[8,9,15]$. This is likely due to the fact that FFR and CFR move in opposite directions from resting conditions to hyperaemia: FFR decreases and becomes more abnormal, while CFR increases and becomes more normal. Hence, the combination of an abnormal FFR and normal $P_{\mathrm{d}} / P_{\mathrm{a}}$ may occur on the basis of a normal $P_{\mathrm{d}} / P_{\mathrm{a}}$ value that decreases to abnormal FFR values at hyperaemia due to a large increase in coronary flow with a normal CFR. Such stenoses are considered non-flow-limiting, and likely have excellent clinical outcomes when managed medically $[8,9,15,17,18]$. On the other hand, an abnormal $P_{\mathrm{d}} / P_{\mathrm{a}}$ may occur in combination with a normal FFR when a stenosis may coexist with an exhausted CFR as a result of compensatory vasodilation during resting conditions to maintain resting perfusion. The abnormal $P_{\mathrm{d}} / P_{\mathrm{a}}$ may only decrease to normal FFR values due to the limited increase in coronary flow from baseline to hyperaemia. The optimal management of vessels exhibiting this haemodynamic pattern remains a matter of debate [19]. Since myocardial function thrives on coronary flow and not on perfusion pressure, reductions in distal coronary perfusion, however, should not be associated with impaired myocardial function as long as adequate coronary flow is present [20]. This is supported by a recent randomised study documenting excellent clinical outcomes when magnetic resonance 
imaging-defined perfusion deficits were used to guide coronary intervention [18]. Since myocardial function and clinical outcomes are determined by coronary flow independent of coronary pressure [8, 9, 15, 20], and $P_{\mathrm{d}} / P_{\mathrm{a}}$ has better agreement with invasively measured coronary flow than FFR [3], this may provide an explanation why $P_{\mathrm{d}} / P_{\mathrm{a}}$ has superior prognostic value when there is disagreement with FFR.

\section{Comparison with previous FFR studies}

Importantly, our observation that there was no difference in clinical outcomes between FFR-positive and FFR-negative cases might wrongly be interpreted to be in contrast with the findings from FAME (Fractional Flow Reserve Versus Angiography for Multivessel Evaluation) and FAME II [16, 21]. FAME compared FFR versus angiography for guidance of revascularisation and documented superior clinical outcome using a FFR-guided revascularisation strategy. FAME II compared PCI + optimal medical therapy (OMT) versus OMT for treatment of FFR-positive lesions and documented superior clinical outcome for lesions treated with PCI+OMT. It is important to note that the average FFR in FFR-positive stenosis in FAME was $0.60 \pm 0.14$ [21]. In FAME II, the average FFR in FFRpositive stenoses amounted to $0.68 \pm 0.10$ in the medical therapy group and $0.68 \pm 0.15$ in the PCI group [16]. Hence, the stenoses included in the FAME studies were physiologically much more severe than those encountered typically in contemporary daily practice. Most importantly, among these severe stenoses in the FAME II study, around $60 \%$ of lesions in the medical therapy group did not require any coronary intervention within 2 years after initial deferral from revascularisation-a result that is consistent up to 5-year follow-up. The present study involves true physiologically intermediate stenoses as typically encountered in daily practice (Tab. 1 ; $[2,10,11,18]$ ). This is relevant for the observed differences in clinical outcomes compared with the FAME studies: FFR reflects a risk continuum, where risk for adverse events becomes higher with decreasing FFR [22]. This risk continuum for FFR was also noted in the Cox regression analysis for FFR in the present study. However, the FAME II trial showed that the benefit of revascularisation was supreme in vessels with FFR $<0.65$. Similarly, a recent patient-level meta-analysis on the prognostic value of FFR identified an optimal 0.67 FFR threshold for clinical outcomes [22]. These findings support the lack of a difference in MACE between FFR $\leq 0.80$ and $>0.80$ in the present study, where, similar to daily clinical practice, most FFR values in FFR-positive stenoses were $>0.70$ and therefore above the FFR thresholds associated with impaired clinical outcomes.

\section{Limitations}

The relatively small sample size limits the statistical power and the strength of the conclusions, especially for the discordant groups. We used intracoronary administration of $20-40 \mu \mathrm{g}$ of adenosine, which remains a subject for debate. However, it has been documented that the observed FFR is already within 0.02 of its minimal value at intracoronary adenosine dosages above $23 \mu \mathrm{g}$ [23]. Since the test/retest repeatability of FFR itself has a standard deviation of 0.02 [24], FFR differences $<0.02$ are smaller than the variability of the FFR measurement itself and, therefore, are considered clinically irrelevant [23]. It is therefore unlikely that the dose of adenosine has interfered with the results in the present study. This is supported by the limited differences in FFR values derived from lowdose intracoronary versus intravenous adenosine administration [25], and by the fact that such small differences in FFR between intravenous and intracoronary adenosine were documented to be clinically irrelevant in the DEFER trial [26]. Additionally, this study is limited by the assessment of adverse events at long-term follow-up partly performed by telephone contact. Such an approach is sensitive towards patient recall bias, which may result in under-reporting of adverse events. Nonetheless, the long-term MACE rates reported in the present study are comparable with those reported in contemporary studies using FFR guidance [26, 27].

\section{Conclusion}

The present study documents that $P_{\mathrm{d}} / P_{\mathrm{a}}$ demonstrates a continuous and independent relationship with subsequent long-term clinical outcomes which is at least equivalent to that of FFR, and suggests that $P_{\mathrm{d}} / P_{\mathrm{a}}$ exceeds FFR as a risk stratification tool at its contemporary cut-off value.

Acknowledgements We gratefully acknowledge F. van der Wal, RN, J.E. Brouwer, RN, and S.H. Oortwijn, RN, for their extensive effort in collecting the follow-up data, as well as the nursing staff of the cardiac catheterisation laboratory (Head: Edith Hilkemeijer) for their skilled assistance in acquiring the data.

Funding Grant support. This work was supported by the European Community's Seventh Framework Programme (FP7/2007-2013) (grant agreement no. 224495), and by grants from the Dutch Heart Foundation (2006B186 and D96.020). The funding sources had no role in the study design or writing of the manuscript.

Conflict of interest G.W.M. Wijntjens is partly supported by a research fellowship grant from Philips-Volcano Corporation. T.P. van de Hoef, M. Meuwissen, M. Echavarría-Pinto and J.J. Piek have served as speakers at educational events organised by St Jude Medical, Boston Scientific, and Volcano Corporation, manufacturers of sensor-equipped coronary guide wires. T. Murai, V.E. Stegehuis, K.T. Koch, S.A. Chamuleau, 
M. Voskuil, R.J. de Winter and J.G.P. Tijssen declare that they have no competing interests.

Open Access This article is licensed under a Creative Commons Attribution 4.0 International License, which permits use, sharing, adaptation, distribution and reproduction in any medium or format, as long as you give appropriate credit to the original author(s) and the source, provide a link to the Creative Commons licence, and indicate if changes were made. The images or other third party material in this article are included in the article's Creative Commons licence, unless indicated otherwise in a credit line to the material. If material is not included in the article's Creative Commons licence and your intended use is not permitted by statutory regulation or exceeds the permitted use, you will need to obtain permission directly from the copyright holder. To view a copy of this licence, visit http://creativecommons.org/licenses/by/4.0/.

\section{References}

1. Sen S, Escaned J, Malik IS, et al. Development and validation of a new adenosine-independent index of stenosis severity from coronary wave-intensity analysis: results of the ADVISE (ADenosine Vasodilator Independent Stenosis Evaluation) study. J Am Coll Cardiol. 2012;59:1392-402.

2. Jeremias A, Maehara A, Généreux P, et al. Multicenter core laboratorycomparison of theinstantaneous wave-freeratio and resting Pd/Pa with fractional flow reserve: the RESOLVE study. JAm Coll Cardiol. 2014;63:1253-61.

3. Echavarría-Pinto $M$, van de Hoef TP, van Lavieren MA, et al. Combining baseline distal-to-aortic pressure ratio and fractional flow reserve in the assessment of coronary stenosis severity. JACC Cardiovasc Interv. 2015;8:1681-91.

4. Hwang D, Jeon K-H, Lee JM, et al. Diagnostic performance of resting and hyperemic invasive physiological indices to define myocardial ischemia: validation with (13)Nammonia positron emission tomography. JACC Cardiovasc Interv. 2017;10:751-60.

5. de Waard GA, Danad I, Petraco R, et al. Fractional flow reserve, instantaneous wave-free ratio, and resting $\mathrm{Pd} / \mathrm{Pa}$ compared with [15O]H2O positron emission tomography myocardial perfusion imaging: a PACIFIC trial sub-study. Eur Heart J. 2018;39:4072-81.

6. Petraco R, van de Hoef TP, Nijjer S, et al. Baseline instantaneous wave-free ratio as a pressure-only estimation of underlying coronary flow reserve: results of the JUSTIFYCFR Study (Joined Coronary Pressure and Flow Analysis to Determine Diagnostic Characteristics of Basal and Hyperemic Indices of Functional Lesion Severity-Coronary Flow Reserve). Circ Cardiovasc Interv. 2014;7:492-502.

7. Cook CM, Jeremias A, Petraco R, et al. Fractional flow reserve/instantaneous wave-free ratio discordance in angiographically intermediate coronary stenoses: an analysis using Doppler-derived coronary flow measurements. JACC Cardiovasc Interv. 2017;10:2514-24.

8. van de Hoef TP, van Lavieren MA, Damman P, et al. Physiological basis and long-term clinical outcome of discordance between fractional flow reserve and coronary flow velocity reserve in coronary stenoses of intermediate severity. Circ Cardiovasc Interv. 2014;7:301-11.

9. Lee JM, Jung J-H, Hwang D, et al. Coronary flow reserve and microcirculatory resistance in patients with intermediate coronary stenosis. JAm Coll Cardiol. 2016;67:1158-69.
10. Davies JE, Sen S, Dehbi H-M, et al. Use of the instantaneous wave-free ratio or fractional flow reserve in PCI. N Engl J Med. 2017;376:1824-34.

11. Götberg M, Christiansen EH, Gudmundsdottir IJ, et al. Instantaneous wave-free ratio versus fractional flow reserve to guide PCI. NEngl J Med. 2017;376:1813-23.

12. Meuwissen M, Siebes M, Chamuleau SAJ, et al. Hyperemic stenosis resistance index for evaluation of functional coronarylesion severity. Circulation. 2002;106:441-6.

13. Meuwissen M, Chamuleau SAJ, Siebes M, et al. The prognostic value of combined intracoronary pressure and blood flow velocity measurements after deferral of percutaneous coronary intervention. Catheter Cardiovasc Interv. 2008;71:291-7.

14. Chamuleau SAJ, Tio RA, De Cock CC, et al. Prognostic value of coronaryblood flow velocity and myocardial perfusion in intermediate coronarynarrowings and multivessel disease. JAm Coll Cardiol. 2002;39:852-8.

15. van de Hoef TP, Echavarría-Pinto M, van Lavieren MA, et al. Diagnostic and prognostic implications of coronary flow capacity: a comprehensive cross-modality physiological concept in ischemic heart disease. JACC Cardiovasc Interv. 2015;8:1670-80.

16. De Bruyne B, Fearon WF, Pijls NHJ, et al. Fractional flow reserve-guided PCI for stable coronary artery disease. NEngl J Med. 2014;371:1208-17.

17. Murthy VL, Naya M, Foster CR, et al. Improved cardiac risk assessment with noninvasive measures of coronary flow reserve. Circulation. 2011;124:2215-24.

18. Nagel E, Greenwood JP, McCann GP, et al. Magnetic resonance perfusion or fractional flow reserve in coronary disease. NEnglJ Med. 2019;380:2418-28.

19. van Lavieren MA, van de Hoef TP, Sjauw KD, et al. How should I treat a patient with refractory angina and a single stenosis with normal FFR but abnormal CFR? EuroIntervention. 2015;11:125-8.

20. Smalling RW, Kelley K, Kirkeeide RL, Fisher DJ. Regional myocardial function is not affected by severe coronary depressurization provided coronaryblood flow is maintained. JAm Coll Cardiol. 1985;5:948-55.

21. Tonino PAL, De Bruyne B, Pijls NHJ, et al. Fractional flow reserve versus angiography for guideing percutaneous coronary intervention. NEnglJ Med. 2009;360:213-24.

22. Johnson NP, Tóth GG, Lai D, et al. Prognostic value of fractional flow reserve: linking physiologic severity to clinical outcomes. J Am Coll Cardiol. 2014;64:1641-54.

23. Adjedj J, Toth GG, Johnson NP, et al. Intracoronary adenosine: dose-response relationship with hyperemia. JACC Cardiovasc Interv. 2015;8:1422-30.

24. Johnson NP, Jeremias A, ZimmermannFM, etal. Continuum of vasodilator stress from rest to contrast medium to adenosinehyperemiafor fractionalflowreserveassessment. JACC Cardiovasc Interv. 2016;9:757-67.

25. Rigattieri S, Biondi Zoccai G, Sciahbasi A, et al. Meta-analysis of head-to-head comparison of intracoronary versus intravenous adenosine for the assessment of fractional flow reserve. Am J Cardiol. 2017;120:563-8.

26. Bech GJ, De Bruyne B, Pijls NH, et al. Fractional flow reserve to determine the appropriateness of angioplasty in moderate coronary stenosis: a randomized trial. Circulation. 2001;103:2928-34.

27. Li J, Elrashidi MY, Flammer AJ, et al. Long-term outcomes of fractional flow reserve-guided vs. angiographyguided percutaneous coronary intervention in contemporary practice. Eur Heart J. 2013;34:1375-83. 